\section{Hacia una educación superior inclusiva en Colombia}

\section{Towards an Inclusive Higher Education in Colombia}

\author{
Para um ensino superior \\ includente na Colômbia
}

\section{Resumen}

En este artículo se analizan concepciones teórico-conceptuales básicas que subyacen a la educación superior inclusiva, en el marco de los Lineamientos de la Política de Educación Superior Inclusiva del Ministerio de Educación Nacional de Colombia (MEN). Además, se plantean los desafíos que deben asumir las instituciones de educación superior (IES) en la identificación de barreras de aprendizaje de su población estudiantil y la promoción de estrategias que garanticen la accesibilidad, permanencia, pertinencia académica y la graduación. Esta situación lleva a las IES a reflexionar y repensarse a partir de los procesos de autoevaluación institucional para crear estrategias participativas que promuevan la gestión inclusiva en un sistema educativo equitativo, pertinente y de calidad.

\section{Palabras clave}

Educación superior inclusiva, índice de inclusión (IIES), acceso, permanencia, graduación, pertinencia.

\section{Abstract}

This article analyzes basic theoretical and conceptual ideas that underlie an inclusive higher education, within the framework of the Policy Guidelines on Inclusive Higher Education proposed by the Ministry of National Education in 2013. It also examines the challenges that higher education institutions must face when identifying the learning barriers of their diverse student populations and promoting strategies that would ensure access, attendance, academic pertinence, and graduation. This situation makes higher education institutions reflect and rethink themselves based on processes of institutional self-evaluation, in order to create participatory strategies that promote inclusive management in a fair, relevant, and quality education system.

\section{Keywords}

Inclusive higher education, index for inclusion (IIES), access, retention, graduation, pertinence.

Fecha de recepción: febrero 10 de 2016 Fecha de aprobación: marzo 27 de 2016

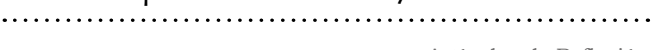
Artículos de Reflexión Pedagogia y Saberes No. 45 Universidad Pedagógica Nacional Facultad de Educación. 2016. pp. 41-52

\section{Sandra Lucia Arizabaleta Domínguez Andrés Felipe Ochoa Cubillos ${ }^{* *}$}

Profesora e investigadora de la Fundación Universitaria Católica Lumen Gentium, magister en Educación Superior de la Universidad Santiago de Cali. Correo electrónico: sarizabaleta@unicatolica.edu.co

* Profesor e investigador de la Fundación Universitaria CatólicaLumen Gentium Magister en Administración de la Universidad Nacional de Colombia. Correo electrónico: afochoa@unicatolica. edu.co 


\section{Resumo}

Neste artigo analisam-se conceições teórico-conceptuais fundamentais, as quais encontram-se no âmago da educação superior includente, no marco dos Lineamentos da Política para a Educação Superior Includente do Ministério de Educação Nacional da Colômbia (MEN). Além disso, propõem-se os desafios que devem assumir as Instituições de Educação Profissional (IEs) na identificação de limites na aprendizagem de sua população estudantil e na promoção de estratégias que garantem o ingresso, permanência, pertinência acadêmica e formatura. Tal situação faz com que as IEs reflitam e pensem a partir dos processos de auto-avaliação institucional com o propósito de criar estratégias de participação que promovam a gestão inclusiva em um sistema educacional equitativo, pertinente e de qualidade.

Palavras-chave

Educação profissional includente, índice de inclusão (IIES), acesso, permanência, formatura, pertinência.

\section{Introducción}

L a educación inclusiva se refiere a la gestión, transformación y adaptación de las instituciones de educación superior (IEs) a las diferentes barreras de aprendizaje y participación de la población estudiantil, con el fin de diseñar procesos de formación inclusivos en los programas académicos, y que estos propendan por la formación de los maestros inclusivos; generar procesos de investigación frente al tema de educación inclusiva y calidad educativa con enfoque inclusivo. Blanco (1999) afirma que la educación inclusiva implica una visión diferente de la educación común basada en la heterogeneidad y no en la homogeneidad, es decir, las IEs deben potenciar y valorar la diversidad cultural de su población estudiantil para promover su participación como sujetos de derechos.

Es necesario abordar y comprender el concepto de educación superior inclusiva y sus dimensiones, para evitar caer en falsas interpretaciones, como sucede respecto a los conceptos de inclusión educativa y educación inclusiva. En este sentido, la inclusión educativa promueve la identificación de las barreras de aprendizaje y la participación de un determinado grupo social con su respectiva situación de aprendizaje en las aulas (Ministerio de Educación Nacional, MEN, 2013). Esta es una educación pensada para una minoría de la población estudiantil. La educación inclusiva incorpora los derechos constitucionales a las políticas y estrategias promulgadas por el MEN para delimitar su objeto y reflexionar sobre el proceso de enseñanza-aprendizaje-evaluación para los diferentes grupos poblacionales, identificar barreras de aprendizaje en las IEs y garantizar la atención a la diversidad. La educación inclusiva dista de la inclusión educativa, al incluir todos los estudiantes en aulas diversas e inclusivas (Stainback \& Stainback, 1999), con el apoyo de la gestión directiva, administrativa, financiera, académica y comunitaria de las IES colombianas.
Al respecto, Fayad afirma que hay un giro lingüístico y conceptual importante cuando se habla de inclusividad:

Porque inclusivo [no incluyente] se refiere a una inclusión en diferentes órdenes, donde diversas formas de incluir logran consolidar y darle forma a la capacidad de que "eso que falta" entre sin el uso de formas hegemónicas o fuerzas que no permiten el desarrollo de lo propio o que nieguen las posibilidades para ese proceso. (2010, p. 2)

Colombia es considerado un país pluriétnico y pluricultural; su diversidad de grupos poblacionales acceden a la educación superior para transformar su vida, su familia y fomentar el desarrollo de las regiones. Parte de la problemática de la educación superior en Colombia se relaciona con: a) dificultades de la población vulnerable y diversa (los grupos étnicos, víctimas del conflicto armado, personas en situación de discapacidad, población ROM, LGTBI, habitantes de frontera, campesinos, entre otros), para acceder a la educación superior, y b) los altos porcentajes de deserción y fracaso estudiantil, que afectan la permanencia y graduación de los estudiantes. De acuerdo al reporte de cobertura o accesibilidad del MEN respecto a deserción y graduación de la educación superior, en el 2012 la tasa de cobertura para acceder a la educación superior a nivel nacional fue del 42,3\%; la tasa de deserción, de 45,3\%, y la tasa de graduación de 33,6 \% (MEN, 2013).

En este sentido, las IEs en Colombia, de cara a un escenario de posconflicto o construcción de paz, deben asumir con responsabilidad social la formación de los estudiantes, desde grupos étnicos, víctimas del conflicto armado, personas en situación de discapacidad, población ROM, LGTBI, habitantes de frontera, campesinos, entre otros, para crear ambientes de aprendizaje inclusivos con impacto social.

Para promover la gestión inclusiva en la educación superior en el país, el MEN dio a conocer a partir de septiembre de 2013, a través de Viceministerio de 
Educación Superior, los Lineamientos de la política de educación superior inclusiva, con el objetivo de fomentar una educación de calidad para todos, respetando y valorando sus diferencias.

El objetivo del artículo es abordar las concepciones teórico-conceptuales en torno al debe ser de la educación superior inclusiva, en el marco de la política de educación superior inclusiva propuesta en el 2013 por el MEN, como también los desafíos que deben asumir las IEs para identificar y superar las barreras de aprendizaje de su población estudiantil y promover estrategias que garanticen su accesibilidad, permanencia, pertinencia y graduación (Sánchez, 1999, 2003).

El Acuerdo de lo superior 2034, propuesto por el Consejo Nacional de Educación Superior (CESU), considera que uno de los pilares para proyectar el sistema educativo superior al 2034 es la educación inclusiva acompañada de las estrategias que desarrollan las IES para garantizar el reconocimiento por la diversidad cultural como aval para incrementar las tasas de accesibilidad, permanencia y graduación de su población estudiantil y disminuir las altas tasas de deserción que tiene la educación superior en Colombia (MEN y CESU, 2014).

El tema se desarrolla a través del planteamiento y la discusión de desafíos que deben asumir las IEs para promover una educación inclusiva equitativa, que permita ofrecer educación de calidad a todas y todos los estudiantes.

\section{Desarrollo de la gestión inclusiva}

El desarrollo de la gestión inclusiva en las IEs requiere asumir con liderazgo tres desafíos: a) sensibilización de la comunidad educativa sobre el concepto de educación inclusiva en el contexto universitario, en el marco de la política de educación superior inclusiva del MEN (2013); b) construcción del índice de inclusión de las IEs como una herramienta que permite construir un diagnóstico sobre la gestión inclusiva de la universidad, y c) diseño e implementación de una política de educación superior inclusiva que se articule a los factores y características de los lineamientos de acreditación del Consejo Nacional de Acreditación (CNA).

\section{Sensibilización de la comunidad educativa sobre el concepto de educación inclusiva en el contexto universitario en el marco de la política de educación superior inclusiva del MEN (2013)}

Hablar de educación superior inclusiva en Colombia remite a la universidad del siglo XXI a revisar su gestión inclusiva como principio rector para luchar contra la exclusión social. La educación superior en Colombia debe ser el laboratorio social propicio para pensar las dinámicas de inclusión y construir sociedades inclusivas. En este sentido, la educación superior en Colombia presenta falencias que se evidencian a través de las estadísticas develadas por el sistema de prevención y análisis de la deserción escolar sobre las IES (MEN, 2013), donde, por ejemplo, para el Valle del Cauca la tasa de cobertura fue de $34,7 \%$, la tasa de deserción $48,8 \%$ y la tasa de graduación 30,5\%, mientras que a nivel nacional fueron de 42,3 \%; 45,3 \% y 33,6\%, respectivamente. En el Valle del Cauca las cifras sobre la calidad de la educación superior no son alentadoras, pues presentan menor tasa de cobertura, mayor tasa de deserción por cohorte y menor tasa de graduación con respecto al promedio nacional.

Las IES en Colombia deben promover en su cultura organizacional una educación inclusiva desde la gestión directiva, administrativa, financiera, académica y comunitaria, como una oportunidad para lograr identificar y superar las barreras de aprendizaje de sus estudiantes y promover la participación de todos y todas, desde su condición étnica, social, política, económica, cultural, lingüística, física, religiosa y geográfica.

Durante la Conferencia Regional de América Latina y el Caribe (CRES, 2008), se reflexionó sobre el concepto de educación superior y se dimensionó su razón de ser, definiéndola como:

... un derecho humano y un bien social. Los Estados tienen el deber fundamental de garantizar este derecho. Los Estados, las sociedades nacionales y las comunidades académicas deben ser quienes definan los principios básicos en los cuales se fundamenta la formación de los ciudadanos y velar porque ella sea pertinente y de calidad. El carácter de bien público social [...] se reafirma en la medida que el acceso a ella sea un derecho real de todos los ciudadanos. (p.5) 
En este marco, la educación para todas y todos debe asumirse como un derecho humano, según lo estipula la Constitución Política de Colombia de 1991, que plantea:

La educación es un derecho de la persona y un servicio público que tiene una función social; con ella se busca el acceso al conocimiento, a la ciencia, a la técnica, y a los demás bienes y valores de la cultura. La educación formará al colombiano en el respeto a los Derechos Humanos (DDHH), a la paz y a la democracia; y en la práctica del trabajo y la recreación, para el mejoramiento cultural, científico, tecnológico y para la protección del ambiente. (Artículo 67)

Toda IEs colombiana, pública o privada, necesita un equipo líder o un grupo de investigación que interprete y comprenda el concepto de educación superior inclusiva a la luz de sus procesos de docencia, investigación, proyección social, bienestar institucional y su estructura y gestión administrativa y financiera, pues la errada interpretación y comprensión conlleva a que se desvíen o focalicen los esfuerzos, de entidades gubernamentales y no gubernamentales, en el apoyo a ciertos grupos sociales, y se propicien la exclusión de otros grupos. Se evidencian errores conceptuales al confundir la educación inclusiva con inclusión social, inclusión educativa, integración, necesidades educativas especiales, con necesidades educativas diversas, y estas, a su vez, con barreras para el aprendizaje.

En los Lineamientos de la política en educación superior inclusiva (2013, p. 24), se explicita la dicotomía conceptual que existe, debido a que la inclusión educativa promueve la adaptación del estudiante al sistema educativo y no al contrario.

El MEN proyecta la educación inclusiva como la estrategia para promover e implementar el proceso de identificación y superación de las barreras de aprendizaje de la población estudiantil, caracterizándolas, categorizándolas, generando proyectos institucionales que promuevan el acceso, la permanencia y la graduación de los estudiantes, construyendo una política educativa inclusiva fundamentada en los DDHH, valorando la diversidad y la participación para fomentar la inclusión social en la universidad (Ginè, 2003 y Meléndez, 2003).

La inclusión educativa dista de la educación inclusiva, en la medida en que la primera promueve la inclusión de ciertos grupos sociales en el sistema educativo. Esto significa pensar y posibilitar la atención de cierta población al sistema educativo, como el efecto generado con la incorporación del concepto de integración, propio de los sistemas educativos internacionales y nacionales de los años 80 y 90 , que se estructuró en el marco de las necesidades educativas especiales (NEE), para la población en situación de discapacidad, que incluyó a unos y excluyó a otros.

Por lo tanto, la educación inclusiva no debe visibilizarse como un objetivo puntual, ni una herramienta o un proceso en sí misma. Ainscow (2001) afirma que la educación inclusiva implica reestructurar cultura, políticas y prácticas de las IE para garantizar la atención a la diversidad y la participación. Los primeros trabajos sobre educación inclusiva se centraron en la inclusión de estudiantes en situación de discapacidad en el sistema educativo regular del Distrito de Woodstock, en Canadá, a través de los esfuerzos del catedrático universitario, Gordon Porter, padre de la educación inclusiva. Porter (1997) afirma que la educación inclusiva es un proceso de transformación de los sistemas educativos convencionales, para brindar una educación con pertinencia a todos los estudiantes, con el fin de levantar las barreras de aprendizaje y lograr su participación.

Porter (1997) identificó tres factores que permiten construir instituciones educativas, programas y aulas inclusivas:

1. Realizar un diagnóstico y análisis en el liderazgo de la política de inclusión, administración e implementación del programa inclusivo.

2. Realizar una descripción de las necesidades del programa para implementar un plan de formación para maestros inclusivos.

3. Implementar los lineamentos de las estrategias que brindan apoyo para el docente de clase que enseña una clase inclusiva, incluyendo estrategias de desarrollo de personal, equipos de pares para resolver problemas, estrategias de instrucción y currículo inclusivos, así como una instrucción a niveles múltiples. La creación de programas educativos inclusivos está ligada a la creación de una educación de calidad para todos los estudiantes.

Los principios de los derechos humanos aprobados en la Convención de 1989 de Naciones Unidas sobre los Derechos del Niño y en la Declaración y Marco de Acción de Salamanca de 1994 de la Unesco (1994), contribuyen a robustecer el concepto de educación inclusiva. Su propósito ayuda a fomentar el respeto por el derecho a la educación para estudiantes con cualquier barrera para el aprendizaje, de manera que se faciliten los espacios para un diálogo de saberes entre los grupos sociales que llegan a las IES, públicas y privadas, con el fin de intercambiar 
saberes y valorar su condición étnica, social, política, económica, cultural, lingüística, física y geográfica, entre otras.

Un significativo aporte al concepto de educación inclusiva lo realizaron Booth y Ainscow (2002), a través de la construcción del índice de inclusión en las escuelas de Canadá e Inglaterra para promover la inclusión. Al respecto, Booth y Ainscow (2000), en su estudio "Index for Inclusion: Developing Learning and Participation in Schools", desarrollaron un instrumento para la evaluación de la gestión inclusiva en las instituciones educativas. Además, crearon un Centro de Estudios para la Educación Inclusiva (CSEI), con el objetivo de crear conciencia sobre la educación inclusiva en los centros educativos.

El instrumento se aplicó en 26 instituciones educativas de Inglaterra, con impactantes resultados sobre la cultura, las políticas y prácticas de una educación inclusiva. Surgieron proyectos de transformación curricular y oferta de programas nuevos para las instituciones. La metodología se fundamentó en la investigación-acción, que se apoyó en un conjunto detallado de indicadores y de preguntas, que han de ser analizadas en profundidad para identificar la situación actual de las instituciones educativas, así como sus posibilidades para avanzar hacia una mayor inclusión. Booth y Ainscow recomendaron para futuras investigaciones que en América Latina y el Caribe el índice se adapte y cambie de acuerdo con las circunstancias locales, siempre que permanezca el espíritu central de su proceso: revisión, consulta, recopilación de información y plan de desarrollo de una institución educativa inclusiva, que ayude a levantar las barreras para el aprendizaje y promueva la participación de la comunidad educativa diversa.

Otro aporte para la construcción del concepto de educación inclusiva fue realizado por Sandoval et al. (2002), a través de la construcción de una guía para la evaluación y mejora de la educación inclusiva en los centros educativos de Madrid, España. El INDEX FoR INCLUSION se ajustó al contexto educativo español con base en la traducción al castellano realizada por la Oficina Regional de la Unesco para América Latina y el Caribe (Orealc). Esta adaptación permitió a los investigadores dimensionar la educación inclusiva en estos centros educativos, desde tres pilares fundamentales (Unesco, 2008):

- Desarrollar prácticas de enseñanza, aprendizaje y evaluación inclusivas.

- Elaborar políticas inclusivas.

- Crear culturas inclusivas.
Uno de los retos de la educación superior inclusiva del siglo XXI consiste en reducir las brechas entre los individuos, poblaciones, regiones e instituciones. Ante este reto, el MEN implementa la política de educación superior inclusiva como principio rector, que debe incorporarse en los procesos educativos universitarios a través de una filosofía de la inclusión. En este sentido, la educación superior inclusiva se convierte en una estrategia para la búsqueda de la inclusión social, con el fin de crear y recrear ambientes de aprendizaje inclusivos para la población en situación de discapacidad, grupos étnicos, campesinos, víctimas del conflicto armado, habitantes de frontera o población en cualquier situación de vulnerabilidad de sus derechos. En esta dirección, se visibiliza la educación inclusiva como el camino hacia el futuro, en la cuadragésima octava reunión de la Conferencia Internacional de Educación (CIE), que tuvo lugar en Ginebra del 25 al 28 de noviembre de 2008.

El MEN y el Centro de Innovación y Desarrollo (CID) (2007) iniciaron un estudio sobre educación inclusiva para la construcción de una política que promueva la educación superior inclusiva en Colombia. El estudio fue realizado a 127 IES colombianas y evidenció un incremento en la tasa de accesibilidad a la educación superior de la población diversa, en un $908 \%$ entre el 2007 y el 2011. El MEN inició un proceso de reflexión sobre la integración y las necesidades educativas especiales con relación a la exclusión de otros grupos sociales, como los étnicos. Ante dicha problemática, en el año 2011 propuso abordar las necesidades educativas diversas, NED, como un cambio de paradigma, para incluir a otros grupos poblacionales en el sistema educativo. Se inició un camino para valorar y potenciar la diversidad. Para el año 2012, el MEN continuó la discusión por el término necesidades, que hacía alusión a una patologización de la población diversa; se inició la construcción conceptual de la educación inclusiva para el país, en aras de identificar las barreras para el aprendizaje y la participación de todos los estudiantes de los diferentes niveles del sistema educativo colombiano y se construyeron los "Lineamientos de la política de educación superior inclusiva”, publicados el 30 de septiembre de 2013.

En el 2012, el MEN convocó a las IEs para que a través de la construcción del índice de inclusión social de los programas contribuyeran a participar con proyectos de educación inclusiva que generaran transformaciones curriculares en los programas establecidos, con la posibilidad de ofrecer nuevos programas tanto a la luz de la formación del maestro inclusivo como de la diversidad. 
La figura 1 muestra los avances en los estudios del MEN, con relación a la educación superior inclusiva desde el 2007 con proyección al 2034.

Figura 1. Evolución del concepto de Educación Inclusiva.

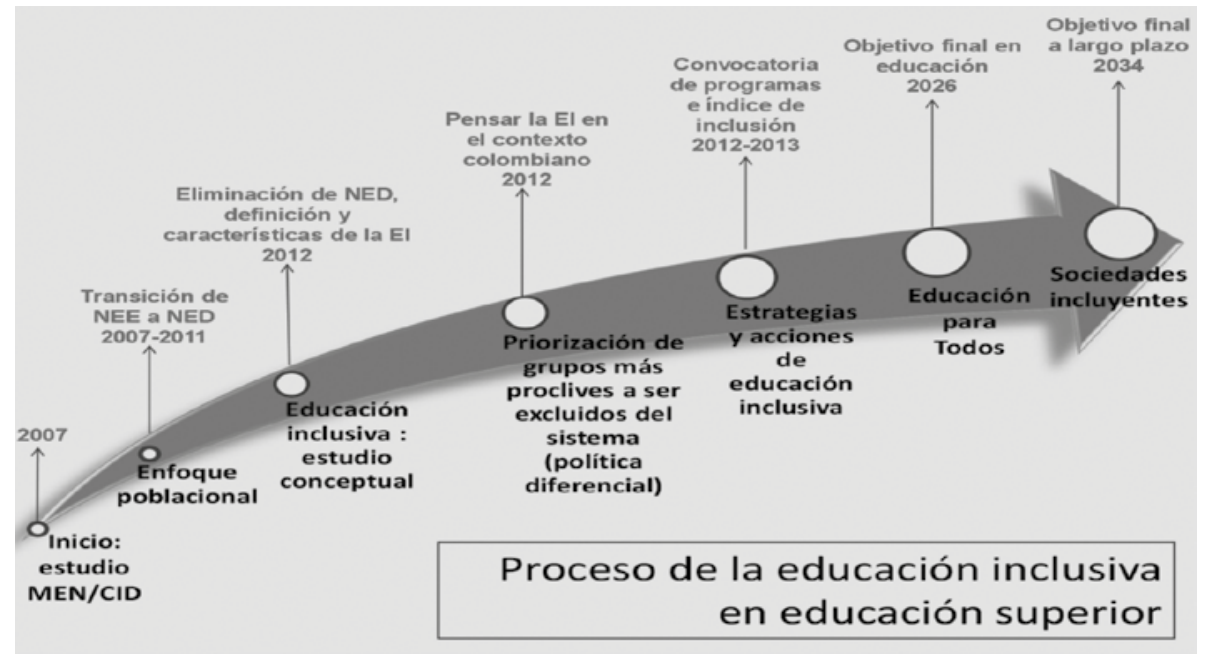

Fuente: Lineamientos de la Política sobre la Educación Superior Inclusiva, MEN, 2013.

De acuerdo a los aportes de Ainscow y Miles (2008) y Acedo (2008), el paradigma de educación inclusiva debe estar sustentado en la pertinencia, participación, equidad, diversidad, interculturalidad y calidad (véase la figura 2).

Figura 2. Características de la Educación Inclusiva.

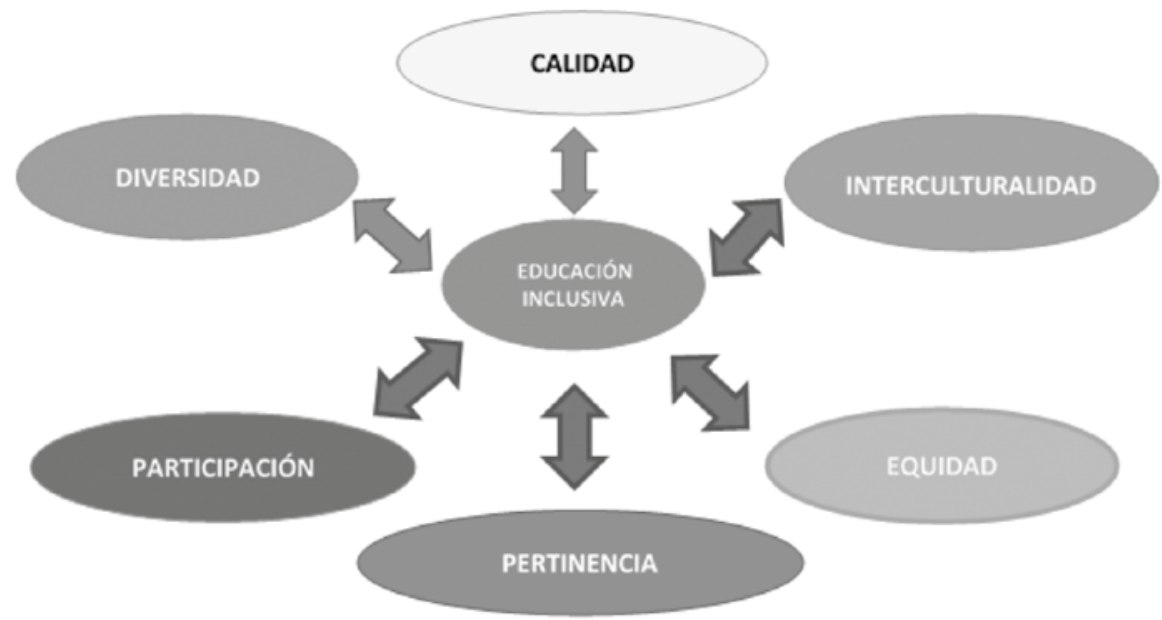

Fuente: Lineamientos de la Política sobre la Educación Superior Inclusiva, MEN, 2013

En el cuadro 1 se describe la relación existente entre las seis características del concepto de educación inclusiva y el contexto universitario colombiano. 
Cuadro 1. Relación entre las características que le dan sentido a

la educación inclusiva y el contexto universitario.

\begin{tabular}{|c|c|}
\hline CARACTERISTICA & APORTES AL CONTEXTO UNIVERSITARIO COLOMBIANO \\
\hline Pertinencia & $\begin{array}{l}\text { Capacidad instalada que tienen las IES para responder a las } \\
\text { necesidades del contexto y generar proyectos de impacto social. }\end{array}$ \\
\hline Participación & $\begin{array}{l}\text { Garantizar la vinculación social de cada uno de los estudiantes al } \\
\text { espacio universitario, valorando su condición étnica, social, cultural, } \\
\text { política, económica, sexual, lingüística, física y geográfica. }\end{array}$ \\
\hline Diversidad & $\begin{array}{l}\text { Hace alusión a la riqueza cultural existente en los diferentes grupos sociales } \\
\text { de nuestro país. Las IEs deben identificarla como la oportunidad que tienen de } \\
\text { fomentar el diálogo de saberes y de rescatar la riqueza de la identidad cultural, } \\
\text { como también las necesidades de los grupos poblacionales colombianos. }\end{array}$ \\
\hline Interculturalidad & $\begin{array}{l}\text { Según los Lineamientos de la política sobre la educación superior inclusiva } \\
\text { (2013), es el conjunto de relaciones entre diferentes grupos culturales } \\
\text { que conduce a un proceso dialógico de constante transformación, } \\
\text { interacción, diálogo y aprendizaje de los diferentes saberes culturales en } \\
\text { el marco del respeto, valorando y rescatando su identidad cultural. }\end{array}$ \\
\hline Equidad & $\begin{array}{l}\text { La universidad debe reconocer la diversidad estudiantil teniendo en } \\
\text { cuenta su caracterización, categorización, diagnóstico y oportunidades } \\
\text { para brindar una educación de calidad para todos los estudiantes. } \\
\text { Además, generar estrategias que garanticen la accesibilidad, permanencia } \\
\text { y graduación de los estudiantes de diferentes grupos sociales. }\end{array}$ \\
\hline Calidad & $\begin{array}{l}\text { Se articula con el seguimiento de la gestión inclusiva ante procesos de } \\
\text { acreditación institucional, acreditación de alta calidad y autoevaluación } \\
\text { de los programas y la formación del maestro para brindar ambientes de } \\
\text { aprendizaje inclusivos y propender por una educación de calidad. }\end{array}$ \\
\hline
\end{tabular}

Fuente: Lineamientos de la política sobre la educación superior inclusiva, MEN, 2013.

Para dar continuidad a la construcción del concepto de educación inclusiva en el contexto universitario, el MEN propone en los Lineamientos de la política de la educación superior inclusiva (2013), cuatro retos para promover en las IEs públicas y privadas la gestión inclusiva, a través de la apropiación, la implementación, el seguimiento y la evaluación de una política de educación superior inclusiva para el país. Los retos son:

- Generar procesos académicos inclusivos.

- Contar con docentes inclusivos, es decir formados en educación inclusiva.

- Promover espacios de investigación, innovación social, de creación artística y cultural con enfoque de educación inclusiva.

- Construiry consolidar una estructura administrativa y financiera que sustente las estrategias y acciones de la educación superior inclusiva.
- Diseñar e implementar una política de educación superior inclusiva institucional.

Dichos retos buscan que las IEs hagan una profunda y rigurosa reflexión alrededor de problemáticas del contexto universitario como la deserción, y a su vez, analicen cómo influyen en la calidad de la educación superior en Colombia. En esta línea, el Consejo Nacional de Educación Superior, CESU, describe en el documento Acuerdo por lo superior 2034, una propuesta de política pública para la excelencia de la educación superior en Colombia, en el escenario de la paz. En él aparece el concepto de educación inclusiva como uno de los ejes estratégicos para estructurar y proyectar el sistema de educación superior al 2034 desde sus estrategias de accesibilidad, permanencia y graduación de todos los estudiantes. En este acuerdo del 2014 se concibe la educación inclusiva como la capacidad de potenciar y valorar la diversidad, promover el respeto a la diferencia y garantizar la participación de la comunidad estudiantil en una estructura intercultural de los procesos educativos. 
Construcción del Î́ndice de Inclusión de la Institución de Educación Superior (IIES) como una herramienta que permite construir un diagnóstico sobre la gestión inclusiva de la universidad

Como ha señalado Vaughan (2002), el Índice de inclusión, desde su lanzamiento en marzo del año 2000 , se ha traducido e implementado en numerosas instituciones educativas de Alemania, Australia, Finlandia, Noruega, Portugal, Rumania, y se pretende traducirlo próximamente al chino para probarlo en instituciones educativas de Hong Kong, al árabe, al francés (Québec) y al maltés. La sede central de la Unesco en París ha financiado diferentes estudios sobre cómo se podría desarrollar un índice adaptado en IES de Brasil, Sudáfrica e India. Algunas IES del Estado de Nueva Yorky de Connecticut han expresado un gran interés en llevar a cabo pruebas del Índice de esas regiones del norte, para brindar una educación de calidad para todos con ambientes de aprendizaje inclusivos.

Los resultados analizados en el Reino Unido muestran que el Índice es considerado un documento profundamente inclusivo y hay evidencia de su influencia positiva en este tema que se refleja desde las declaraciones de los ministros de Educación del
Gobierno, hasta los administradores locales de educación en las autoridades locales de educación y los docentes de las IEs.

En Colombia se está llevando a cabo la prueba piloto con el MEN para la construcción del Índice de inclusión en la educación superior, como una herramienta que les permitirá a las IES analizar su proceso de educación inclusiva a través de la aplicación de un instrumento con 21 indicadores articulados a los diez factores propuestos por el CNA. Está segunda fase es vital para construir un diagnóstico sobre la gestión inclusiva de la universidad.

\section{Diseño e implementación de una política de educación superior inclusiva que se articule a los factores y características de los lineamientos de acreditación del CNA}

En el cuadro 2 se muestra una propuesta para articular los indicadores de la gestión inclusiva en las IES y los procesos de acreditación de alta calidad y autoevaluación de los programas a los diez factores y características propuestos por el CNA (2014), con el fin de promover la equidad y la participación activa de la población diversa en las IES, en aras de brindar una educación pertinente y de calidad.

Cuadro 2. Articulación entre los factores del CNA, características e

indicadores para promover la gestión inclusiva en las IES.

\begin{tabular}{|c|c|c|}
\hline FACTOR DEL CNA & CARACTERÍSTICA & INDICADOR \\
\hline \multirow{3}{*}{$\begin{array}{l}\text { Misión, proyecto } \\
\text { institucional y programa }\end{array}$} & $\begin{array}{l}\text { Misión y proyecto } \\
\text { educativo institucional }\end{array}$ & $\begin{array}{l}\text { La misión, la visión y el proyecto educativo de } \\
\text { la universidad promueven la gestión inclusiva. }\end{array}$ \\
\hline & Proyecto educativo del programa & El proyecto educativo del programa es inclusivo. \\
\hline & $\begin{array}{l}\text { Relevancia y pertinencia } \\
\text { social del programa }\end{array}$ & $\begin{array}{l}\text { El programa responde a las } \\
\text { necesidades del entorno. }\end{array}$ \\
\hline \multirow{4}{*}{ Estudiantes } & Accesibilidad de los estudiantes & $\begin{array}{l}\text { La IES crea estrategias para el ingreso } \\
\text { de la población diversa. }\end{array}$ \\
\hline & $\begin{array}{l}\text { Estudiantes admitidos y } \\
\text { capacidad institucional }\end{array}$ & $\begin{array}{l}\text { La IEs incluye a la población diversa y cuenta } \\
\text { con la capacidad instalada para ofrecer una } \\
\text { educación inclusiva, integral y de calidad. }\end{array}$ \\
\hline & $\begin{array}{l}\text { Participación en programas } \\
\text { de formación integral }\end{array}$ & $\begin{array}{l}\text { La IES cuenta con una caracterización y un } \\
\text { diagnóstico del estudiante que ingresa para } \\
\text { implementar un programa de formación } \\
\text { integral de acuerdo con la identificación } \\
\text { de barreras para el aprendizaje. }\end{array}$ \\
\hline & Reglamento estudiantil & $\begin{array}{l}\text { El reglamento estudiantil evidencia procesos } \\
\text { de inclusión para la población diversa. }\end{array}$ \\
\hline
\end{tabular}




\begin{tabular}{|c|c|c|}
\hline \multirow{8}{*}{ Profesores } & $\begin{array}{l}\text { Selección y vinculación } \\
\text { de profesores }\end{array}$ & La IES selecciona y vincula a profesores inclusivos. \\
\hline & Estatuto profesoral & $\begin{array}{l}\text { El reglamento profesoral cuenta con } \\
\text { el perfil del maestro inclusivo. }\end{array}$ \\
\hline & $\begin{array}{l}\text { Nivel de formación de los } \\
\text { profesores y experiencia }\end{array}$ & $\begin{array}{l}\text { El profesor a través de su nivel de formación } \\
\text { disciplinar, pedagógica y didáctica y experiencia } \\
\text { investigativa promueve una educación inclusiva. }\end{array}$ \\
\hline & Desarrollo profesoral & $\begin{array}{l}\text { El profesor es constantemente formado } \\
\text { para promover una educación inclusiva. }\end{array}$ \\
\hline & $\begin{array}{l}\text { Estímulos a la docencia, la } \\
\text { investigación, la creación artística } \\
\text { y cultural, la proyección social y la } \\
\text { extensión e internacionalización }\end{array}$ & $\begin{array}{l}\text { El docente investigador e investigador } \\
\text { docente cuenta con estímulos para } \\
\text { promover proyectos de educación inclusiva } \\
\text { a nivel nacional e internacional. }\end{array}$ \\
\hline & Producción intelectual & $\begin{array}{l}\text { El profesor es reconocido por su producción } \\
\text { intelectual en educación inclusiva y su impacto social. }\end{array}$ \\
\hline & Remuneración por méritos & $\begin{array}{l}\text { El profesor cuenta con una remuneración por } \\
\text { méritos, al promover una educación inclusiva } \\
\text { a través de la formulación, el desarrollo y la } \\
\text { evaluación de proyectos de investigación. }\end{array}$ \\
\hline & Evaluación de profesores & $\begin{array}{l}\text { La evaluación de los profesores incluye indicadores } \\
\text { que promueven la educación inclusiva. }\end{array}$ \\
\hline \multirow{8}{*}{ Procesos académicos } & Integralidad del currículo & $\begin{array}{l}\text { El currículo integra el diseño, la implementación } \\
\text { y la evaluación de un proceso de formación, } \\
\text { que le apuesta al desarrollo de valores, } \\
\text { actitudes y aptitudes de la población diversa. }\end{array}$ \\
\hline & Flexibilidad del currículo & $\begin{array}{l}\text { El currículo es flexible y se adapta a las } \\
\text { condiciones particulares de la población diversa. }\end{array}$ \\
\hline & Interdisciplinariedad & $\begin{array}{l}\text { El currículo promueve el diálogo de saberes } \\
\text { disciplinares, entre contextos y culturas. }\end{array}$ \\
\hline & $\begin{array}{l}\text { Metodología de enseñanza } \\
\text { y aprendizaje }\end{array}$ & $\begin{array}{l}\text { El programa cuenta con pedagogías } \\
\text { y didácticas flexibles para garantizar } \\
\text { ambientes de aprendizaje inclusivos. }\end{array}$ \\
\hline & $\begin{array}{l}\text { Sistemas de evaluación } \\
\text { de los estudiantes }\end{array}$ & $\begin{array}{l}\text { El programa cuenta con un proceso } \\
\text { de evaluación inclusivo. }\end{array}$ \\
\hline & $\begin{array}{l}\text { Evaluación y autorregulación } \\
\text { de los programas }\end{array}$ & $\begin{array}{l}\text { El programa lleva a cabo un proceso de } \\
\text { autoevaluación donde le hace seguimiento a la gestión } \\
\text { inclusiva con su respectivo plan de mejoramiento. }\end{array}$ \\
\hline & Extensión o proyección social & $\begin{array}{l}\text { El programa cuenta con una oferta de } \\
\text { formación en la identificación de barreras } \\
\text { para el aprendizaje para el sector externo. }\end{array}$ \\
\hline & Recursos bibliográficos & $\begin{array}{l}\text { El programa cuenta con recursos bibliográficos } \\
\text { de acuerdo a las barreras de aprendizaje } \\
\text { identificadas en los estudiantes. }\end{array}$ \\
\hline
\end{tabular}




\begin{tabular}{|c|c|c|}
\hline FACTOR DEL CNA & CARACTERÍSTICA & INDICADOR \\
\hline \multirow[t]{2}{*}{ Procesos académicos } & $\begin{array}{l}\text { Recursos informáticos } \\
\text { y de comunicación }\end{array}$ & $\begin{array}{l}\text { El programa cuenta con los recursos informáticos } \\
\text { y de comunicación para suplir las barreras para } \\
\text { el aprendizaje de la población estudiantil. }\end{array}$ \\
\hline & Recursos de apoyo docente & $\begin{array}{l}\text { El programa cuenta con los recursos necesarios } \\
\text { para que el docente oriente a la población diversa. }\end{array}$ \\
\hline Internacionalización & $\begin{array}{l}\text { Inserción del programa } \\
\text { en contextos académicos } \\
\text { nacionales e internacionales }\end{array}$ & $\begin{array}{l}\text { La IES tiene convenios nacionales } \\
\text { e internacionales, que promuevan } \\
\text { la gestión inclusiva. }\end{array}$ \\
\hline \multirow{2}{*}{$\begin{array}{l}\text { Investigación, innovación, } \\
\text { creación artística y cultural }\end{array}$} & $\begin{array}{l}\text { Formación para la } \\
\text { investigación, innovación, } \\
\text { creación artística y cultural }\end{array}$ & $\begin{array}{l}\text { La IES promueve la formación en } \\
\text { investigación en educación inclusiva. }\end{array}$ \\
\hline & $\begin{array}{l}\text { Compromiso con la } \\
\text { investigación, innovación, } \\
\text { creación artística y cultural }\end{array}$ & $\begin{array}{l}\text { La IES promueve la formulación, la } \\
\text { implementación y la evaluación de proyectos } \\
\text { de investigación en educación inclusiva. }\end{array}$ \\
\hline \multirow{2}{*}{ Bienestar institucional } & $\begin{array}{l}\text { Políticas, programas y servicios } \\
\text { de bienestar universitario }\end{array}$ & $\begin{array}{l}\text { La IES diseña e implementa políticas, } \\
\text { programas, servicios de bienestar } \\
\text { universitario para la población diversa. }\end{array}$ \\
\hline & Permanencia estudiantil & $\begin{array}{l}\text { La IES diseña e implementa políticas } \\
\text { y programas que promueven la } \\
\text { permanencia de los estudiantes. }\end{array}$ \\
\hline \multirow{3}{*}{$\begin{array}{l}\text { Organización, } \\
\text { administración y gestión }\end{array}$} & $\begin{array}{l}\text { Organización, administración } \\
\text { y gestión del programa }\end{array}$ & $\begin{array}{l}\text { La organización, administración y gestión del } \\
\text { programa promueve el desarrollo de la gestión } \\
\text { inclusiva en articulación con las funciones } \\
\text { sustantivas de docencia, investigación, creación } \\
\text { artística, cultural, extensión y proyección social. }\end{array}$ \\
\hline & $\begin{array}{l}\text { Sistemas de comunicación } \\
\text { e información }\end{array}$ & $\begin{array}{l}\text { El programa cuenta con sistemas de comunicación } \\
\text { e información, accesibles a la población diversa. }\end{array}$ \\
\hline & Dirección del programa & $\begin{array}{l}\text { El programa cuenta con un líder que } \\
\text { promueva la gestión inclusiva. }\end{array}$ \\
\hline \multirow{3}{*}{$\begin{array}{l}\text { Impacto de los graduados } \\
\text { en el medio }\end{array}$} & Seguimiento de los graduados & $\begin{array}{l}\text { La IES cuenta con un programa de } \\
\text { seguimiento a los graduados. }\end{array}$ \\
\hline & \multirow{2}{*}{$\begin{array}{l}\text { Impacto de los graduados en } \\
\text { el medio social y académico }\end{array}$} & $\begin{array}{l}\text { La IES cuenta con un programa de } \\
\text { vinculación laboral para los graduados. }\end{array}$ \\
\hline & & $\begin{array}{l}\text { La IES mide el impacto de los graduados } \\
\text { en el medio social y académico. }\end{array}$ \\
\hline \multirow{3}{*}{$\begin{array}{l}\text { Recursos físicos } \\
\text { y financieros }\end{array}$} & Recursos físicos e infraestructura & $\begin{array}{l}\text { La IES cuenta con la infraestructura de } \\
\text { acuerdo a la Norma Técnica Colombiana } \\
\text { (NTC) para el desarrollo de las funciones } \\
\text { sustantivas de la población diversa. }\end{array}$ \\
\hline & Presupuesto del programa & $\begin{array}{l}\text { El programa dispone de un presupuesto } \\
\text { que promueva las funciones sustantivas } \\
\text { hacia la gestión inclusiva. }\end{array}$ \\
\hline & Administración de recursos & $\begin{array}{l}\text { La administración de los recursos físicos } \\
\text { y financieros de la IES promueve la } \\
\text { gestión inclusiva de los programas. }\end{array}$ \\
\hline
\end{tabular}

Fuente: CNA (2013). 


\section{Conclusiones}

El concepto de educación superior inclusiva es reciente en Colombia y por ende, su comprensión e implementación requiere de un debido proceso de sensibilización hacia la comunidad educativa, para promover la cultura inclusiva desde las funciones sustantivas de proyección social, docencia e investigación, con el fin de proyectar una educación de calidad y equidad en la universidad, a través de los siguientes desafíos:

- Sensibilización de la comunidad educativa sobre el concepto de educación inclusiva en el contexto universitario en el marco de la política de educación superior inclusiva del MEN (2013).

- Construcción del índice de inclusión de las IES como una herramienta que permite construir un diagnóstico sobre la gestión inclusiva de la universidad.

- Diseño e implementación de una política de educación superior inclusiva que se articule a los factores y características de los lineamientos de acreditación del CNA.

En sus esbozos, la educación inclusiva nació de la educación especial, que se ofrecía a unos cuantos grupos poblacionales. Más adelante, se reflexionó sobre la oportunidad de incluir a los estudiantes en situación de discapacidad en las aulas regulares, que se implementó en Canadá a través de unas nuevas políticas educativas que obtuvieron excelentes resultados, gracias a los aportes de Porter, quien se convirtió en el padre de la educación inclusiva. Aunque la visión de Porter sobre la educación inclusiva fue de corto alcance, pues el deseo de incluir a la población estudiantil en situación de discapacidad en las aulas regulares excluía a otros grupos poblacionales, fue a partir de su propuesta que se empezó a hablar de aulas inclusivas (Stainback \& Stainback, 1999; Tlistone, 2003).

En el contexto educativo colombiano se han evidenciado dinámicas sociales como el desplazamiento y el conflicto armado, que han promovido un camino hacia la inclusión de varios grupos poblaciones en la educación superior. Esto se evidencia en un estudio realizado en el año 2007 por el MEN y el Centro de Investigaciones para el desarrollo de la Universidad Nacional de Colombia (CID): de 242 estudiantes de diferentes grupos poblacionales que ingresaron en el 2002 pasaron a 2439, en el año 2007. En cinco años se incrementó en un $908 \%$ la población diversa en 127 IES del país. Este reto remite a las IEs a la transformación del currículo, las prácticas pedagógicas y didácticas, los proyectos de investigación, la proyección social, el bienestar institucional, su gestión administrativa, directiva y financiera. Es imperativo que cada IEs en Colombia analice los niveles de accesibilidad, permanencia, deserción y graduación de la población diversa para promover la gestión inclusiva, en la que no basta con formar maestros inclusivos, se requiere de voluntad política y recursos financieros para lograrlo.

Las IES deben emprender un camino hacia una educación inclusiva a través de la construcción del índice de inclusión como una herramienta que permite diagnosticar y conocer el estado de su gestión inclusiva, en aras de construir unos planes de mejoramiento y mantenimiento para fortalecer el proceso de autoevaluación de los programas alineados al de Acreditación de Alta Calidad y lograr desarrollar procesos inclusivos como:

- Crear y ofertar programas de educación superior inclusivos.

- Formar docentes inclusivos.

- Construir políticas institucionales que propendan por una educación superior inclusiva.

- Generar proyectos de educación superior inclusiva para participar en la convocatoria del MEN y así promover la investigación social.

- Participar en la revisión y los ajustes de los Lineamientos de la política de educación superior inclusiva de septiembre de 2013.

\section{Referencias bibliográficas}

Acedo, C. (2008). Educación inclusiva. Perspectivas, 38(1).

Ainscow, M. (2001). Desarrollo de escuelas inclusivas: ideas, propuestas y experiencias para mejorar las instituciones escolares (vol. 84). España: Narcea.

Ainscow, M. y Miles, S. (2008). Por una educación para todos que sea inclusiva. ¿Hacia dónde vamos ahora? Perspectivas, 38(1), 17-44.

Blanco, R. (1999). Hacia una escuela para todos y con todos. Boletín 48.

Booth, T. y Ainscow, M. (2002). Index for Inclusion: Developing learning and participation in schools. Center for Studies on Inclusive Education.

Conferencia Regional de Educación Superior. (2008). Declaración final de la Conferencia Regional de Educación Superior en América Latina y el Caribe. Autor. 
Fayad, S. J. (2010). La educación superior inclusiva, concepciones, tipologías, construcción de comunidades de vida y comunidades de sentido universitario para todas y todos. Cali: Universidad del Valle.

Ginè, C. (2003). Inclusión y sistema educativo. Ponencia presentada en el III Congreso La Atención a la Diversidad en el Sistema Educativo. (U.d. Comunidad, Recopilador) Salamanca.

Meléndez, L. (2003). Atención a la diversidad en el contexto educativo costarricense. División de Desarrollo Curricular / MEP (mimeo).

Ministerio de Educación Nacional y Consejo Nacional de Educación Superior. (2014). Acuerdo por lo Superior 2034: propuesta de política pública para la excelencia de la educación superior en Colombia en el escenario de la paz. Autor.

Ministerio de Educación Nacional y Centro de Innovación y Desarrollo del Tecnológico de Antioquia. (2007). Programa de educación inclusiva con calidad. Autor.

Ministerio de Educación Nacional. (2013). Caracterización de los estudiantes y diagnóstico de deserción: departamentos de Cauca, Huila, Nariño, Putumayo y Valle del Cauca. Bogotá: Autor.

Ministerio de Educación Nacional. (2013). Lineamientos de la política en educación superior inclusiva. Bogotá: Autor.
Porter, G. (1997). Elementos críticos para escuelas inclusivas. En S. Pijl, C. Meijer \& S. Hegerty. Educación inclusiva y agenda global (pp. 68-81). Londres.

Sánchez, A. (1999). El reto de educar en una sociedad multicultural y desigual. En A. Sánchez et ál., Los desafíos de la educación especial en el umbral de siglo XXI (pp. 61-90). Almería: Servicio de Publicaciones de la Universidad.

Sánchez, A. (2003). Educación inclusiva: una escuela para todos. Aljibe.

Sánchez, A. (2003). Los estudiantes con discapacidad en una escuela para todos. Conferencia.

Sandoval, M.; López, M.; Miquel, E.; Durán, D.; Giné, C. y Echeita, G. (2002). Index for Inclusion: Una guía para la evaluación y mejora de la educación inclusiva. Contextos Educativos: Revista de Educación, 5, 227-238.

Stainback, S. y Stainback, W. (1999). Aulas inclusivas. Madrid: Narcea.

Tlistone, C. (2003). Promoción y desarrollo de prácticas educativas inclusivas. Nueva York.

Unesco. (1994). Informe final Conferencia Mundial sobre Necesidades Educativas Especiales. Acceso y calidad. París: Unesco/MEC.

Unesco. (2008). Educación inclusiva: el camino hacia el futuro. XLVIII Conferencia Internacional de Educación., Ginebra.

Vaughan, M. (2002). An index for inclusion. European Journal of Special Needs Education, 17(2), 197-201. 